\title{
Percepción del duelo en jóvenes universitarios de la carrera de Psicología del Instituto de Ciencias de la Salud
}

\author{
Perception of grief in youth undergraduate students of the Psychology career from the Instituto \\ de Ciencias de la Salud
Jorge G. Escobar-Torres ${ }^{a}$, Yetzai Trujillo-Cornejo ${ }^{b}$, Santos N. Herrera-Mijangos ${ }^{c}$, Dayana Luna-Reyes ${ }^{d}$, Itzia Cázares-Palacios ${ }^{e}$, Flor Gil-Bernal ${ }^{f}$ y Edith Gómez-Gamero ${ }^{g}$

\begin{abstract}
:
This study aimed to explore the perception of grief in students. The method used was based on Grounded Theory. The sample was a total of 10 youth undergraduate students of the Psychology career from the Instituto de Ciencias de la Salud among 20-25 years old who have experienced one or more grief experience. The Discourse Analysis was the methodical strategy for analyzing the answers of the participants.

The results showed that the process of grief has been experimented at least once in life of the majority of people and every person has lived in different way, depending of internal and external factors. The emotions and feelings that participants experimented due a significant lost are sadness, anger, disappointment and resentment. Likewise, it was found that when a person who has lived a process of grief it is easier for themselves to put in the place of others persons in a similar situation.

Finally, in conclusion the grief could be experienced by any person and caused for different situations such as death of a familiar, a breakup of couple, the change of house or school or the loss of something material.
\end{abstract}

Keywords:

Grief, emotional dependence, undergraduate student, emotions

\section{Resumen:}

Esta investigación tuvo como objetivo conocer la percepción de los estudiantes sobre el duelo. El método utilizado se basó en la teoría fundamentada. Se utilizó una muestra de 10 estudiantes de la Licenciatura en Psicología del Instituto de Ciencias de la Salud con edades entre los 20 y 25 años de edad que hayan experimentado una o más experiencias de duelo. El análisis del discurso fue la estrategia metodológica empleada para analizar las repuestas de los participantes.

Los resultados obtenidos en la presente investigación indican que el proceso de duelo se ha experimentado al menos una vez en la vida de la mayoría de las personas y cada persona lo vive de diferente manera, dependiendo de factores internos y externos. Las emociones y sentimientos que se enfrentan las participantes ante una pérdida significativa generalmente son de tristeza, enojo, decepción y resentimiento. Asimismo se encontró que cuando una persona ha vivenciado un proceso de duelo le es más fácil ponerse en el lugar del otro ante una situación similar.

Finalmente se concluye que el duelo se puede presentar en cualquier persona y puede ser causado por distintas situaciones como la muerte de un familiar, una ruptura de pareja, el cambio de casa o escuela o bien la pérdida de algo material.

\section{Palabras Clave:}

Duelo, dependencia emocional, estudiantes universitarios, emociones

\footnotetext{
Autor de Correspondencia, Universidad Autónoma del Estado de Hidalgo, http://orcid.org/0000-0002-9598-8781, Email: jorgegonzaloescobar@gmail.com

b Universidad Autónoma del Estado de Hidalgo, https://orcid.org/0000-0001-9535-5397, Email: yetzai.tru@ gmail.com

c Universidad Autónoma del Estado de Hidalgo, https://orcid.org/0000-0001-6567-0986, Email: psicologonoe@yahoo.com.mx

${ }^{d}$ Universidad Autónoma del Estado de Hidalgo, https://orcid.org/0000-0001-9687-2521, Email: dayis2902@ gmail.com

e Universidad Autónoma del Estado de Hidalgo, https://orcid.org/0000-0002-0498-3820, Email: itzia_cazares@uaeh.edu.mx

f Universidad Autónoma del Estado de Hidalgo, https://orcid.org/0000-0001-7756-3125, Email: florerari@ yahoo.com

g Universidad Autónoma del Estado de Hidalgo, https://orcid.org/0000-0002-7699-9995, Email: edgomo2@gmail.com
} 


\section{Introducción}

El proceso de duelo siempre ha estado presente a lo largo de la vida de un ser humano, no distingue sexo, raza, nivel socioeconómico; en ese sentido toda persona en el mundo en cualquier etapa de su vida puede experimentar un proceso de duelo. Aún con ello, el proceso puede adquirir ciertas particularidades en diferentes lugares del mundo, dependiendo de distintos factores como el contexto, la cultura, la religión, entre otros.

Respecto a las causas activadoras se encuentra que el duelo puede ser originado por distintas situaciones como una ruptura amorosa, la pérdida de un trabajo, el mudarse de casa, la muerte de una mascota, hasta la muerte de un ser querido.

En cuanto a la definición se encuentra que la palabra duelo proviene del latín "dolus" que indica una respuesta emotiva que puede ser provocada por una pérdida de algo o alguien. Freud en su libro "Duelo y Melancolía" hace hincapié en la reacción que existe ante la pérdida de una persona. Por otro lado, se señala que la melancolía es un tipo de pérdida patológica que presenta el yo de manera que trata de conservar al objeto perdido (1).

Según The National Institutes of Health entre el $10 \%$ y el $20 \%$ de las personas que han vivido una pérdida no son capaces de integrar la experiencia; asimismo se pueden desarrollar problemáticas intensas que son vividas profundamente en el proceso de duelo (4).

El duelo es una circunstancia personal que es inevitable en la perspectiva humana; en ese sentido Muñoz señala que "Desde que el hombre es hombre, se ha sentido mal: sufre. Forma parte de su esencia, como lo hace la muerte que es, entre muchas otras, una de las causas de ese dolor". El modo en que el ser humano ha intentado aliviar este sentimiento ha variado a lo largo del tiempo ( 2 p 2).

Al momento de enfrentar una pérdida Rando et al. consideran que la persona en proceso de duelo tiene afectada la autopercepción de sí y la percepción del mundo; además las personas se enfrentan a una constante preocupación por el futuro, comienzan a cuestionar muchas de las creencias a las cuales están sujetas. Así mismo tienen dificultad para enfocar su atención a la pérdida del objeto o persona, lo que puede desencadenar reacciones emocionales, cognitivas, somáticas y comportamentales (5).
Por otra parte, para Serrano el duelo es considerado como un proceso normal y sería necesario que todos los días éste se elabore. Ello con el objetivo de que las distintas áreas de su vida no presenten un impacto negativo, sino que el trabajo, escuela, familia y amigos sirvan como impulso para el desarrollo (6).

Por otro lado, Sánchez-Aragón y Retana-Franco determinan que existen algunos factores que pueden influir en cómo se presenta un proceso de duelo, por lo que consideran que la elaboración del duelo depende de las creencias y las interpretaciones sobre una pérdida a las que está sujeta cada persona (7).

Finalmente, para Izurieta el duelo de una ruptura amorosa se presenta de manera frecuente en la vida de las personas; la forma en que se lleva a cabo la resolución del duelo es distinta dependiendo de la importancia de la relación amorosa, de manera que la duración e intensidad del duelo pueden provocar diferentes impactos en la calidad de vida de una persona (8).

Por lo tanto, es importante conocer las etapas del duelo; éstas no siempre se presentan con similares características en todas las personas pues varía la intensidad y la duración de cada una de ellas. Por ello es fundamental reconocer cuándo un duelo se vuelve complicado y cuándo se debe buscar ayuda psicológica para poder mejorar la calidad de vida y potenciar el desarrollo de la persona durante el proceso de duelo. Al no elaborarse el duelo, puede iniciarse un duelo patológico lo cual provocaría que la persona que lo sufre no pueda continuar con su vida de manera normal, tendría que acudir a terapia para poder trabajar el duelo, de lo contrario puede presentar conductas desadaptativas.

Por tales razones en este trabajo se busca comprender cuál es la percepción de diez estudiantes sobre lo que consideran duelo, así como conocer las experiencias que ellos mismos o personas cercanas a los participantes han vivido.

\section{Marco Teórico}

Fases

Izurieta logra identificar y distinguir las diferentes fases de duelo, dentro de las cuales se presentan sentimientos de incredulidad, negación y la presencia de un estado de shock, ira, impotencia, frustración y nostalgia. Las fases de duelo identificadas no se presentan en un orden establecido, sino que dependen de las vivencias y recursos que tenga una persona (8). 
De acuerdo a Ríos y Castaño en el proceso del duelo se encuentran 3 subcategorías: el duelo por el entorno (pérdida de tierras), el duelo por un ser querido y el duelo por el cambio de un rol, es decir por la asunción de nuevas responsabilidades (12).

\section{Emociones}

De acuerdo al estudio de Muñoz se determina que el duelo complicado surge cuando no se permite sentir las emociones de tristeza y lo que más promueve al duelo complicado es el sentimiento de culpa (2).

Por su parte Carmona y Bracho señalan que los sentimientos que pueden llevar a complicar el duelo, son los sentimientos de frustración, ansiedad, angustia, incompetencia y la contemplación de su propia muerte (3).

Intervención psicológica

De acuerdo a la investigación de Serrano las personas que padecen el duelo tienden a la búsqueda de implementación de estrategias de intervención, como las consultorías, primeros auxilios psicológicos, capacitación, entre otros, con el fin de paliar el sufrimiento vivido y mejorar su calidad de vida (6).

Por su parte Díaz y Campillo consideran que el uso de la terapia narrativa es útil para las personas que están viviendo un proceso de duelo, dado que ello ayuda a que las personas puedan manejar de mejor manera las situaciones difíciles (16).

Por otro lado, Miaja y Moral, a través de su investigación, han confirmado el significado psicológico de la negación, la ira, la negación, depresión y aceptación de las 5 etapas de duelo que propone Kübler-Ross (17).

Ahora bien, Bermejo y Sánchez encontraron que la mayoría de las personas buscaban apoyo en los grupos de ayuda debido al fallecimiento por enfermedad de familiares. Consideran que la mayor parte de las personas que sufren una pérdida acuden a tratamiento para la salud mental y además ingieren fármacos. En cuanto a las condiciones somáticas, la percepción de las personas con duelo se encontraba exacerbada. Asimismo, se presentaba debilidad muscular y dentro de las emociones aparecen la tristeza, aflicción y miedo (18).

En otro estudio Galindo encuentraron que las emociones más relevantes después de una pérdida amorosa son nostalgia, tristeza, desilusión, rencor, enojo, inseguridad y miedo. De acuerdo a este autor en los hombres surge, ante una ruptura amorosa, el factor de reconciliación debido a creencias culturales de reconquista y solución; mientras que las mujeres presentan un mayor número de conductas depresivas a diferencia de los hombres (10).

\section{Depresión}

Por otra parte, Chaurand et al. observaron una relación leve y moderada entre la depresión y el duelo difícil. No se demostró que existe una disminución a medida que pasa el tiempo, sino que existen variaciones, por lo que algunos de los síntomas se presentan en personas que han sufrido una pérdida entre 6 a 12 meses después. A modo de conclusión Chaurand et al. encontraron que 1 de cada 8 personas que han sufrido una pérdida, en un periodo de entre 6 a 12 meses, tienen complicaciones en su proceso de duelo (11).

\section{Tipos de pérdidas}

En otra investigación Villacieros et al. concluyen que la mayoría de personas que sufren un duelo complicado son las que han perdido a un cónyuge o hijo/a y no han tenido ningún tipo de ayuda (13).

\section{Ruptura amorosa}

De acuerdo a García el proceso de duelo se ha experimentado al menos una vez en la vida de la mayoría de las personas y cada persona lo vive de diferente manera, dependiendo de factores internos y externos. Asimismo, concluye que, si una persona no lleva a cabo su duelo de forma asertiva, puede tener presentar problemas para establecer nuevos vínculos amorosos (9).

Izurieta realiza una investigación para conocer el proceso que se vive tras una ruptura amorosa en jóvenes de 18 a 25 años y encuentra que una de las reacciones más comunes frente a la pérdida, es el dolor (8).

Por otra parte, Sánchez y Martínez encuentran que existen variables como la depresión, emociones de tipo negativo, decepción y suspicacia, las cuales se presentan en diferentes momentos del proceso. Asimismo, determinan como primera etapa del duelo la negociación, después la hostilidad y desesperanza; y al final surge la etapa de la aceptación. Para concluir Sánchez y Martínez consideran que la ruptura amorosa es un tema de interés en diferentes áreas, tanto personales como sociales, ya que es un tema que afecta la vida de cualquier ser humano (14).

De igual manera Espinosa, Salinas y Santillán encontraron que más de la mitad de los estudiantes que 
acuden por ayuda después de pasar una ruptura amorosa son mujeres. En el caso de los hombres, ellos reportan mayor estrés, quizá por la dificultad de reconocimiento en sus emociones. Sugieren que debe existir mayor enfoque en temas como afrontamiento y soluciones de problemas; por ello sugieren el uso de técnicas de corte cognitivoconductual dado que son eficaces para tratar problemas de duelo asociados a la ruptura amorosa (15).

\section{Género}

En cuanto al duelo por pérdida de algún ser querido por el padecimiento de una enfermedad, Miaja y Moral encontraron que la frecuencia de género fue más elevada en las mujeres (17).

Por su lado Moreno y Esparza indican que existe una diferencia significativa en el grupo de mujeres que presentan una aflicción aguda (19).

\section{Objetivo}

Conocer la percepción sobre el duelo e identificar el tipo de duelo que se presenta en jóvenes universitarios de la carrera de Psicología de la Universidad Autónoma del Estado de Hidalgo, a través de entrevistas semisestructuradas.

\section{Metodología}

Los fundamentos epistemológicos de la metodología cualitativa utilizada en la presente investigación son aquellos que radican en la obra de Strauss y Corbin quienes elaboran la Teoría Fundamentada (Grounded Theory); dica teoría ha tomado sustento a partir de los trabajos sobre el pragmatismo de Peirce (1839-1931) y en el interaccionismo simbólico de Mead (1863-1931). Es así que el interaccionismo simbólico señala que "Las acciones humanas se basan en los significados que los actores consideren apropiados; estos significados se derivan de la interacción social con los demás, de modo que los significados son construidos/modificados por los actores a través de interpretaciones y experiencias sociales" (20) (21 p. 8).

\section{Instrumentos para la recolección de información}

Para esta investigación se empleó una entrevista semiestructurada a través de la cual se recabó la información respecto a la opinión y experiencias sobre el duelo en jóvenes universitarios, que pertenecen a la carrera de Psicología.

\section{Muestra}

Se utilizó una muestra no probabilística por conveniencia de 10 estudiantes universitarios de la carrera de Psicología, pertenecientes al estado de Hidalgo; el rango de edad de los participantes fue de 20 a 25 años.

\section{Variables}

Las variables que se emplearon en la presente investigación son el tipo de duelo y percepción del mismo en jóvenes universitarios que pertenecen a la carrera de Psicología.

\section{Procedimiento}

Para la selección de los participantes se requirió que tuvieran una edad mínima de 18 y 25 como máximo y que hubieran sido partícipes de una o más experiencias de duelo a lo largo de su vida; asimismo se requería que fueran estudiantes de la Lic. en Psicología del ICSa.

Se acudieron a los salones en donde toman clase los alumnos para realizar la invitación de manera verbal a participar en las entrevistas. Se mencionó que el objetivo era conocer las percepciones y vivencias en el caso de pérdidas importantes. Los participantes fueron incluidos en la muestra con el sólo requisito de que aceptaran la invitación a participar en la entrevista.

Se determinó que, a fin de contar con el tiempo suficiente para el análisis del material, el tope máximo de personas entrevistadas fueran 10. Los participantes decidieron colaborar de manera voluntaria en las entrevistas individuales realizadas.

Primero se les brindó la información sobre su participación en la entrevista; al comenzar la misma se iniciaba con un saludo y posteriormente las preguntas se iban mencionando y otras iban surgiendo en el momento. Finalmente se agradeció su participación.

\section{Herramientas de análisis}

Mediante el análisis del discurso de cada uno de los participantes se realizó una clasificación para distinguir las opiniones similares a cerca de la percepción del duelo. 


\section{Resultados y discusión}

Para los resultados se realizó una discriminación en el discurso de los participantes para identificar cuáles son las opiniones que más se repiten a lo largo de las entrevistas realizadas. A partir de lo anterior entonces se fueron construyendo las diferentes categorías. Todas las participantes manifestaron algún tipo de pérdidas y las reacciones que presentaron durante el proceso de duelo son parecidas.

Tipos de pérdidas

Existen diferentes tipos de pérdidas y éstas dependen de cada individuo en relación a cuál es el lugar en donde se desenvuelve, el nivel socioeconómico que tienen, la cultura que los rodea, así como de ciertas creencias que tienen sobre sí mismos y el entorno. Un tipo de pérdida expresado por los participantes, sería la muerte de un ser querido como en este caso el abuelo:

"Una pérdida pues es cuándo alguien pierde a algún ser querido, por ejemplo, en mi caso, hace 8 años perdimos a mi abuelo, a mi abuelo materno. Entonces esa es la única pérdida que considero he tenido". ${ }^{1}$

Otra persona relata otro tipo de pérdida como lo es la ruptura amorosa:

"Yo estaba como que muy aferrada a una relación, aunque la otra persona no daba lo mismo ...no daba lo mismo hacia mí. Cuando estábamos juntos, era muy buena la relación, pero cuando no nos veíamos, él no me buscaba. Yo tenía que buscar a esa persona, entonces no era equitativa la relación".²

Así mismo otro participante, expresó la muerte de su abuela como pérdida:

"Pues cuando murió mi abuelita, éramos muy cercanas y pues cuando ya murió, igual. Yo estaba muy chiquita entonces pues siento que me afectó mucho. Yo jugaba con ella casi todos los días después de la escuela; iba a comer a su casa y los fines de semana igual íbamos a su casa"

Otra persona expresa otro tipo de pérdida como lo es el distanciamiento de una mascota:

"Creo que cuando era niña tenía una perrita que quería mucho; entonces apenas me empezaba a acostumbrar a ella y mis papás, o sea no se murió la perrita, sino que la

\footnotetext{
${ }^{1}$ ([Comunicación personal]. 8 de mayo 2019).

2 ([Comunicación personal]. 9 de mayo 2019).
}

regalaron y yo sí me sentía muy triste por ella.... intentaba dormir y me acordaba de la perrita y me ponía a llorar y después cuando despertaba, igual me ponía a llorar; como que me afectó mucho eso".

Otra de las participantes expresó que se puede vivenciar un duelo a través de la pérdida de algo material:

"Lo primero que se me viene la mente fue cuando perdí algo material y yo había ahorrado mucho para comprarlo, entonces siento que eso ha sido como hasta ahorita: bueno lo que se me viene a la mente, lo más significativo: mi celular. Todo lo que me daban mis abuelitos y mis papás y todo, pues lo guardaba y mucho tiempo no me compré nada y pues entonces ya me lo compré y lo perdí luego, luego". ${ }^{3}$

Otra persona relata que se puede vivir un duelo al cambiar de casa y escuela por mudarse de un estado a otro:

"Hace como muchos años, pues sí como nueve, tuve que cambiar de Estado, entonces pues yo vivía en el Estado de México y me tuve que cambiar al Estado de Hidalgo; entonces pues eso me afectó mucho, yo estaba muy triste y pues no quería aceptarlo y pero pues toda mi familia iba a estar acá y no me podía quedar sola allá y aparte siento que me afectó mucho porque yo estaba como en la adolescencia; entonces sí me afectó muchísimo". ${ }^{4}$

\section{Tiempo de duración del duelo}

En cuanto a la duración del proceso de duelo, no hay un tiempo especificado ni definido; cada persona tiene su propio tiempo y esto depende de la importancia o intensidad del lazo que se establece con el objeto o persona amada, así mismo influye la capacidad de redirigir. Las respuestas ubican al duelo entre 5 meses y un año.

\section{Sentimientos/Emociones}

Las emociones que se presentan en cada duelo son distintas, éstas dependen del tipo de pérdida y la manera en la que esta sucedió. Generalmente las personas que atraviesan por una etapa de duelo manifiestan sentimientos y emociones similares. Las emociones que más se han presentado en las entrevistas fueron, tristeza, decepción, resentimiento, enojo tal y como se observa en los siguientes relatos.

\footnotetext{
3 ([Comunicación personal]. 15 de mayo 2019).

${ }^{4}$ ([Comunicación personal]. 12 de mayo 2019).
} 
"Bueno yo perdí a mi familiar a una edad en la que no estaba tan madura como para asimilarlo y saber qué es lo que estaba pasando realmente. Saber que no lo iba a volver a ver fue una tristeza muy grande".

"Pues primero fue, mucha tristeza, decepción, un poco de resentimiento; después se fue calmando mi situación y lo olvidé. Después ya no sentía nada y lo dejé ser".

" Al principio como que no creía que había fallecido, pero pues ya cuando llegamos al lugar donde estaba el velorio pues me empecé a sentir muy triste. Todo eso duró, pues duró un buen rato o sea como ese sentimiento que tenía duró mucho tiempo". 5

\section{Personas de Apoyo}

Para las personas que están atravesando por un proceso de duelo les ayuda que existan personas que pueden apoyarles, a las que le pueden contar cómo se sienten; lo cual es benéfico para que el duelo sea superado de forma más rápida. Algunas de las personas a las que acuden los participantes cuando están atravesando por un duelo, es a la madre o a algún miembro de la familia:

"Sí, mi madre como siempre me escuchaba y me apoyaba y me daba consejos para que yo pudiera salir de esta situación".

"Pues, de mi familia, ellos como que me animaban y me decían que era como normal en los animalitos; o sea que como las personas tenían que morir y así. Y me animaban y trataban como de invitarme al parque 0 hacer actividades en familia para que yo no estuviera todo el día acostada o dormida".

\section{Formas de expresar el dolor}

Para cada persona la manera en cómo externa el dolor ante las personas allegadas es diferente; algunos prefieren llorar, gritar y enojarse ya sea con ellos mismos o con otras personas, mientras que otros prefieren hacerlo de manera solitaria sin expresar lo que verdaderamente sienten o realizando actividades para olvidarse de su dolor. Los participantes informaron que la forma en que pueden expresar su dolor es a partir de aislarse del ambiente en el que se desarrollan, guardarlo para sí mismos, seguir realizando sus actividades cotidianas, hasta llegar a la irritabilidad y enojo hacia otras personas, tal y como se observarse en los siguientes relatos:

\footnotetext{
5 ([Comunicación personal]. 20 de mayo 2019).

6 ([Comunicación personal]. 15 de mayo 2019).
}

"Mi manera de expresar el dolor, fue que no hablaba casi con mis amigos, no salía... lloraba ...no hablaba casi con nadie". 6

"Pues es que no lo expreso o sea yo seguí haciendo mis actividades de todos los días y pues sí me sentía triste. Pero no dejé de hacer lo que hacía normalmente o sea todo siguió normal...pero me guardé todo eso".

"Entonces cuando lo perdí me porté muy grosera con ellos porque yo pues le estaba diciendo que era también su culpa; porque no habían visto y así, entonces siento que sí afecto porque los empecé a tratar muy mal". 7

\section{Cambio en la percepción}

La mayoría de las personas que vivencian una pérdida tiene un cambio en la forma en que perciben el mundo y se perciben así mismos; el perder algo o alguien que es amado puede marcar la vida de un sujeto, la percepción sobre la situación puede verse de manera positiva o negativa, según la persona que lo vive. Los participantes destacan que, a partir de la pérdida, su percepción hacia la vida cambia; de manera en que se tiene que seguir con la vida sin aferrarse a las cosas y ver a la muerte como un proceso normal y como una fuente de mucho aprendizaje. Las sguientes transcripciones dan cuenta de lo anterior:

"Sí, porque ahora sé que una persona no puede cambiar, no puede quitarte algunas cosas. O sea que tú tienes que seguir con tu vida y vivirla feliz, sin estar triste por nadie".

"Sí, también aprendí a que no debo aferrarme a las cosas y que debo seguir con mi vida normal".

"Pues sí, como que ahora acepto más saber que todas las personas que están cerca de mí en algún momento van a morir, ¿no? O hasta yo y ahora siento que lo tomo como un proceso y es algo normal, algo que va a pasar".

\section{Personas cercanas con duelo}

Los seres humanos tendemos a sentir empatía ante situaciones en las que nuestros seres amados sienten dolor, así es más fácil sentirnos identificados cuando alguien está experimentando un duelo si ya hemos vivido algo similar. Cuando una persona cercana atraviesa por un proceso de duelo, los participantes indican que pueden recordar su pérdida y a partir de ella entender el dolor de la otra persona; de modo que la emoción encontrada en el discurso de los participantes fue tristeza:

\footnotetext{
7 ([Comunicación personal]. 4 de mayo 2019).
} 
"Me viene automáticamente un recuerdo de mi abuelo, entonces entiendo su dolor, me pone triste y los entiendo completamente. Les ofrezco mi confianza para escucharlos, para que se desahoguen, para que lloren, para abrazarlos, para que sientan que no están solos y que siempre van a tener a alguien a quien contarle algo". 8

"Pues no sé, es que es muy raro, pero siento que soy una persona muy sentimental y cuando estoy con personas que están en el proceso de una pérdida me siento muy triste; o sea como que me pongo en su lugar y me siento muy triste".

\section{Cambio de la experiencia}

Después de un proceso de duelo generalmente las personas consideran que sí desearían haber cambiado toda la situación que trajo consigo dolor; pero algunas otras personas lo ven como un impulsor para su crecimiento personal y motivador para cumplir objetivos y metas que, de no ser por un momento de dificultad, no se hubieran logrado.

En ese sentido los participantes manifestaron que a partir de su pérdida se obtiene fortaleza, crecimiento emocional y personal, así como un factor que determina su forma de ser en la actualidad. He aquí un ejemplo de este proceso:

"Cada experiencia te vuelve más fuerte tal vez, tienes nuevos conocimientos, [surge] un crecimiento emocional y personal". ${ }^{9}$

\section{Conclusiones}

Se puede concluir que el duelo se puede presentar en cualquier persona y puede ser causado por distintas situaciones como la muerte de un familiar, una ruptura de pareja, el cambio de casa o escuela o bien la pérdida de algo material.

Los resultados obtenidos en la presente investigación concuerdan con los de García quien afirma que el proceso de duelo se ha experimentado al menos una vez en la vida de la mayoría de las personas y cada persona lo vive de diferente manera, dependiendo de factores internos y externos. Asimismo, una persona no lleva a cabo su duelo de forma asertiva, ya que puede presentar problemas para establecer nuevos vínculos; de esa manera la duración del proceso de duelo puede variar de acuerdo a los factores internos y externos. Dicha duración está en relación con el significado que le da la persona a

\footnotetext{
8 ([Comunicación personal]. 2 de mayo 2019).
}

la persona u objeto perdido; así como por el lazo que se mantiene entre ambas personas, por lo que el tiempo que una persona experimenta síntomas y emociones depende de las experiencias previas a la pérdida. Además, existen algunos otros factores que pueden determinar la duración, como el exponerse a objetos que contengan recuerdos sobre la pérdida y pensamientos recurrentes sobre el evento ya que pueden prolongar la duración del proceso (9).

Las emociones y sentimientos que surgieron en los participantes ante una pérdida significativa generalmente son de tristeza, enojo, decepción y resentimiento; estas emociones pueden variar de intensidad y duración, ya que durante el proceso de duelo se presentan distintas fases. En ese sentido, se concuerda con los resultados obtenidos por Muñoz, Galindo y Bermejo y Sánchez Galindo $(2,10,18)$.

Por otra parte, se encontró una concordancia con las investigaciones de Izurieta y Miaja y del Moral dado que no todas las personas entrevistadas presentan todas las fases y la duración de cada una depende de las experiencias previas y el contexto en donde se desarrolla cada individuo $(8,17)$.

Sin importar lo difícil que fue superar una o varias situaciones de duelo en algún momento de la vida, las participantes consideran que haber vivenciado un tipo de pérdida ha servido de forma positiva para ser la persona que son actualmente; al mirar hacia el pasado consideran que dichas pérdidas les ha brindado fortaleza y un crecimiento de índole personal. Asimismo, recuerdan con nostalgia, pero con valentía aquellas vivencias que en alguna etapa de su vida les costó aceptar y asimilar.

Por otra parte, cuando se presenta un proceso de duelo la mayoría de las personas entrevistadas acude con alguien cercano para poder sentir apoyo. En ese sentido se halló una similitud con los hallazgos de Espinosa, Salinas y Santillán, de Bermejo y Sánchez $(15,18)$.

Sin embargo, también se encontró en la presente investigación que mientras para algunas personas es mejor aislarse y no hablar con nadie para poder asimilar las cosas. Ambos casos son positivos para una rápida superación del duelo.

De modo relevante se encontró que cuando una persona ha vivenciado un proceso de duelo le es más fácil ponerse en el lugar del otro ante una situación similar, ya

${ }^{9}$ ([Comunicación personal]. 6 de mayo 2019). 
que tiene conciencia de lo que conlleva una pérdida y todo el proceso al que se pueden enfrentar.

Es importante considerar cuales son los parámetros de normalidad para un proceso de duelo, pues éste se puede complicar y convertirse en un duelo complicado o patológico y de esa manera afectarse la calidad de vida de la persona en distintas áreas de su vida. Los resultados obtenidos en este renglón tienen concordancia con lo realizado por Freud, Rando et al., Chaurand et al. y Villacieros $(1,5,11,13)$.

Por último, cabe mencionar la relevancia de esta investigación en el sentido que aporta una escucha para el derrrotero ineludible para el ser humano: la muerte. La vida emocional en torno al duelo es un proceso que merece ser escuchado e investigado, sobre todo en estas épocas en donde la pandemia del Covid-19 ha movilizado todas las actividades de las personas, las sociedad y gobiernos tal y como señalan Barbancho, Jiménez, Silva y Vialás (22)

Tener un marco de expresión de los afectos y sentimientos vividos en la etapa de duelo constituye un marco para que las personas viviendo dicho proceso movilicen sus capacidades de resiliencia, eviten una prolongada depresión que les podría poner en situación más vulnerable antes las enfermedades, para entonces poder continuar la vida de la mejor manera honrando a los que ya partieron.

Como corolario los autores expresamos nuestro sentir al respecto plasmado en la frase mencionada por un hombre sabio, Andrés Monter, originario de San Juan Solís, Hidalgo:

\section{El verdadero combate,}

no implica ni triunfo ni derrota,

\section{sino el constante atrevimiento}

\section{Referencias}

[1] Freud S, Duelo y melancolía. Obras Completas. Madrid, España. Biblioteca nueva; 1917.

[2] Muñoz M. El efecto del guión de vida sobre el duelo: a propósito de un caso. Rev Digital de Medicina Psicosomática y Psicoterapia 2015; 5(2): 132.

[3] Carmona Z, Bracho C. La muerte, el duelo y el equipo de salud. Rev de salud pública 2008; 2(2): 14-23.

[4] When a loved one dies. Hospice friendly Hospitals Programme 2009 Recuperado a partir de: https://www.hse.ie/eng/services/list/3/acutehospitals/hospitals/cavanmonag han/when-a-loved-one-dies.pdf
[5] Rando T, Doka K, Fleming S, Franco MH, Lobb E, Murray C, Steele R. A Cali to the Field: Complicated Grief in the DSM-V. Omega 2012; 65(4): 251-255.

[6] Serrano Sáenz A, Villancís Puma WG (dir). El efecto del duelo en el desempeño laboral de los colaboradores de recursos humanos de una organización privada de la ciudad de Quito [tesis de licenciatura].[Quito]: Universidad de las Américas; 2017. Recuperado a partir de: http://dspace.udla.edu.ec/handle/33000/6821

[7] Sánchez R, Ratana B. Evaluación tridimensional del duelo amoroso en México. Rev Iberoamericana de Diagnóstico y Evaluación Psicológica 2013; 36(2): 49-69.

[8] Izurieta C. Proceso de elaboración de duelo en rupturas amorosas de jóvenes de 18 a 25 años [Tesis de licenciatura]. [México]: Escuela de Psicología UDLA; 2015. Recuperado a partir de: http://dspace.udla.edu.ec/handle/33000/7275

[9] García D. Narración del duelo en la ruptura amorosa. Ajayu 2014; 12(2): 288-307.

[10] Galindo V. El duelo romántico en la víctima del rompimiento: el papel del apego, las premisas histórico-socio-culturales y la historia romántica [Tesis de licenciatura]. [México]: Universidad Nacional Autónoma de México; 2018.

[11] Chaurand A, Zacarías J, Benítez-Borrego S, Nuñez-Quintero L, Feixas G. Pérdida, depresión y duelo complicado en una muestra mexicana. Rev de Psicología, 2015; 17(2): 42-55.

[12] Ríos Restrepo J, Castaño Gómez SC, Ortiz Sánchez RD (dir). Revisión de relatos frente la experiencia subjetiva del desplazamiento forzado en relación a los cambios, el duelo y las estrategias de afrontamiento [Tesis de licenciatura]. [Antionquia]: Corporación Universitaria Lasallista; 2016. Recuperado a partir de:

http://repository.lasallista.edu.co/dspace/bitstream/10567/2078/1/Desplaza mientoForzado_estrategias_afrontamiento.pdf

[13] Villacieros M, Magaña M, Bermejo JC, Carabias R, Serrano I. Estudio del perfil de una población de personas en duelo complicado que acuden a un centro de escucha de duelo. Med Paliat 2012; 21(3): 91-97.

[14] Sánchez R, Martínez R. Causas y Caracterización de las Etapas del Duelo Romántico. Acta de investigación psicológica 2014; 4(1): 1329-1343. [15] Espinosa V, Salinas J, Santillán C. Incidencia del duelo en la ruptura amorosa en estudiantes universitarios en un Centro de Crisis, Emergencias y Atención al Suicidio (CREAS). Journal of Behavior, Health \& Social Issues 2017; 9(2): 27-35.

[16] Díaz M, Campillo M. Manejo de duelo con terapia narrativa: "Honrar la vida más allá de la muerte". Rev de psicología: Procesos psicológicos y sociales 2016; 1: 7-72.

[17] Miaja M, Moral J. El significado psicológico de las cinco fases del duelo propuestas por Kübler-Ross mediante las redes semánticas naturales. Psicooncología 2013; 10(1): 109-130.

[18] Bermejo J, Sánchez E. La muerte, el duelo y el equipo de salud. Rev de salud pública 2007; 18(2): 82-90.

[19] Moreno J, Esparza E. Estrategias de afrontamiento en la ruptura de pareja. Rev científica y profesional de la Asociación Latinoamericana para la Formación y la Enseñanza de la Psicología 2014; 5(2): 98-109.

[20] Strauss A, Corbin J. Basics of qualitative research: Techniques and procedures for developing grounded theory (2nd ed.). California, Estados Unidos. SAGE Publications; 1998.

[21] Bryant A. Re-grounding grounded theory. JITTA 2002; 4(1): 1-18. [22] Barbancho Ma, Jiménez A, Silva A, Vialás L. Covid-19: Guía para familiares en duelo, Sevilla, España, Col. Ofi. de Psic. de Andalucía Occ, 2020. Recuperado de

http://www.infocoponline.es/pdf/GUIA_PARA_FAMILIARES_EN\%20D UELO_GT\%20Duelo.pdf 